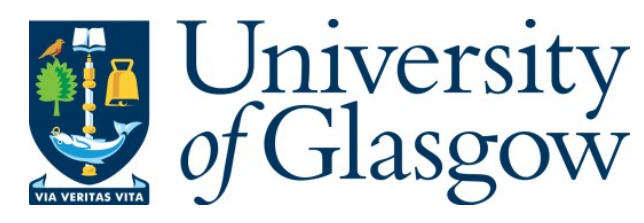

Sheafi, E.M ., and Tanner, K.E. (2014) E ffects of test sample shape and surface production method on the fatigue behaviour of PM M A bone cement. Journal of the M echanical B ehavior of Biomedical M aterials, 29 (1). pp. 91-102. ISSN 1751-6161

Copyright $\odot 2014$ Elsevier

A copy can be downloaded for personal non-commercial research or study, without prior permission or charge

Content must not be changed in any way or reproduced in any format or medium without the formal permission of the copyright holder(s)

When referring to this work, full bibliographic details must be given

http://eprints.gla.ac.uk/85005/

Deposited on: 23 M ay 2014

Enlighten - Research publications by members of the University of Glasgow http://eprints.gla.ac.uk 


\title{
Effects of test sample shape and surface production method on the fatigue behaviour of PMMA bone cement
}

\author{
E. M. Sheafi*, K.E. Tanner
}

\begin{abstract}
There is no consensus over the optimal criterion to define the fatigue life of bone cement in vitro. Fatigue testing samples have been made into various shapes using different surface preparation techniques with little attention being paid to the importance of these variations on the fatigue results. The present study focuses on the effect of test sample shape and surface production method on the fatigue results. The samples were manufactured with two cross sectional shapes: rectangular according to ISO 527 and circular according to ASTM F2118. Each shape was produced using two methods: direct moulding of the cement dough and machining from oversized rods. Testing was performed twice using two different bone cements: SmartSet GHV and DePuy CMW1. At least 10 samples of each category were tested, under fully reversed tension-compression fatigue stress at $\pm 20 \mathrm{MPa}$, to allow for Weibull analysis to compare results. The growth of fatigue cracks was observed by means of the changes in the absorbed energy and apparent modulus. It was found that fatigue crack growth can be altered by the sample shape and production method; however it is also dependent on the chemical composition of the cement. The results revealed that moulded samples, particularly those based on the ASTM F2118 standard, can lead to up to 5.5 times greater fatigue lives compared to the machined samples of the same cement. It is thus essential, when comparing the fatigue results of bone cement, to consider the effect of production method along with the shape of the test sample.
\end{abstract}

\section{Keywords:}

Polymethylmethacrylate, bone cement, fatigue, sample shape, sample surface preparation method, Weibull analysis, absorbed energy

http://dx.doi.org/10.1016/j.jmbbm.2013.08.023

\section{INTRODUCTION}

Polymethylmethacrylate (PMMA) based bone cement has been used in cemented joint arthroplasty since the 1960s. The role of this polymeric substance is to fill the gap between the bone and the implant connecting them together and working as a grout transferring the loads generated from gravity and muscle actions. Fatigue failure of the cement mantle has been considered a major issue that leads to many undesirable consequences. Fundamentally, the key mechanical functions of bone cement reduce after cement failure. Biologically, the fracture of the mantle leads to the detrimental production and spread of PMMA and opacifier particles. The presence of these cement particles increases bone resorption (Quinn et al., 1992; Sabokbar et al., 1996) and leads to various biological responses depending on particle shape and size (Gelb et al., 1994; Mitchell et al., 2003) as well as the type of opacifying agent included (Mitchell et al., 2003; Lezarus et al., 1994; Wang et al., 2005). More importantly, concerns have been raised regarding the likely contribution of this failure in accelerating aseptic loosening, the cause of approximately $75 \%$ of all revisions of both cemented and uncemented total hip replacements [Malchau et al., 2000].
These facts have made it vitally important to investigate variables that control the fatigue behaviour of bone cement. In vitro, much research has been performed on this aspect using various testing methods. The factors such as chemical formulation, storage temperature, viscosity, mixing method and resultant porosity have been examined extensively with variations in results. The inconsistency in the reported results has generated the question as what controls these variations in fatigue behaviour. Lewis and Nyman (2000) examined the literature pointing out that 'a plethora of test conditions have been used' adding that 'many literature parametric studies employed inappropriate statistical methods' and 'these studies have not addressed the issue of possible interactions between the parameters being investigated'. It is widely reported that fatigue life of elements can variously be affected by many design variables including, but not limited to, manufacturing method, cross section and geometry and cyclic deformation mode. Fatigue testing specimens are usually designed and tested in such a way that 'the required test conditions are simulated as closely as possible' (Swanson, 1974). The manufacturing methods can be classified, as in Bhandari (2007), into three categories: casting processes (e.g. mould casting), deformation processes (e.g. extrusion) and material removal or machining processes (e.g. milling). Although casting can be 'one of the easiest methods to convert the raw 
material into finished component' (Bhandari, 2007), the most common method in preparing fatigue specimens is to machine them from non-waisted oversized rods, presuming that the specimen is not overheated or excessively stressed by the machining process (Swanson, 1974). The material removing approach is particularly applicable for specimens with circular sections that are cyclically loaded under various deformation modes including rotating bending (Suresh, 1998). One main aspect that has been reported to relate to the significance of the selection of a particular manufacturing process is the resultant surface roughness and integrity. The greater the deviation from a polished surface the less the material strength since the rougher surfaces provide more local stress concentration sites that enhance the initiation of microcracks which propagate leading to fatigue failures (Mott, 2004). Various standards have been published to help in providing guidelines for preparing specimens and testing the properties of materials such as those published in the ISO and ASTM standards. The ISO standards, for example, include specific standards that describe the procedures to be followed when preparing testing specimens by injection and moulding (e.g. ISO 293) or machining from compression- or injection-moulded rods (e.g. ISO 2818). Despite the consideration that fatigue failures depend essentially on surface conditions where a high fraction of fatigue cracks progress from the surface, it seems there is no specific manufacturing process to provide the 'best' surface quality of a component or specimen for all materials as this can largely be controlled by the properties of the shaped material as well as the manufacturing conditions.

In terms of sample profile, fatigue specimens can be either unnotched (smooth) or notched to produce a stress concentration site in the failure section where the latter is characterised by the elastic stress concentration factor (Dowling, 2007). Specimens are designed with a particular shape of the gauge section providing circular or rectangular cross sectional areas. Review of the literature shows that a typical fatigue life testing specimen generally is divided into three to five regions: the gauge section in the centre of the specimen that has a significantly reduced cross sectional area compared to the other parts of the specimen, two grip ends to be used for fixing the specimen in the testing machine, and two transitional sections between the gauge and the grips through which the cross area is gradually reduced from the grips to the gauge section. The specimens, particularly the circular, can be either solid or hollow as appropriate to represent the real-life conditions. Although the cross sectional shapes are mainly limited to rectangular or circular, the geometries of the gauge and non-gauge sections vary largely depending upon the properties of the material being tested. Generally, two main criteria should be considered when designing fatigue specimens: (1) ensuring the occurrence of the failure in the gauge section and (2) avoiding the presence of stress raisers unless deliberately required (Swanson, 1974). The stress concentrations mentioned in the latter criterion such as notches are included in specimens for special testing requirements as the Compact Tension specimens used in measuring the fatigue crack growth rate within a material.

In addition to specimen production method and profile, the effect of cyclic deformation mode and type should also be considered. A component or specimen might encounter a single direction stress (uniaxial) or interference of two (biaxial) or more (multiaxial) stresses. Fatigue stresses are classified depending upon the position and direction of the applied cyclic load. To assess the fatigue properties of a material, fatigue samples are cyclically loaded by applying axial tension and/or compression, bending or torsion loads as required. Combinations of different stresses such as axial tension and torsion can exist simultaneously. These combined stresses lead to more complex situations as the stresses might differ in phase or frequency or both depending on the sources of cyclic loading (Dowling, 2007). An early study by Gough and Pollard (1935), for instance, proposed an elliptical quadrant criterion to consider the combined effect of, and the relation between, the bending stress and the torsion stress in metals considering their fatigue limits in single load cases. With either mode, one of the following stress types is usually performed in fatigue testing: fully reversed tension-compression, zero-tension or tensiontension stress. The selection of the appropriate stress type is made depending on the conditions that a material would encounter in 'real life'.

Considering the application of the previous procedures for bone cement, it is significant to identify which sample preparation techniques and stress regimes have specifically been used and reported. A review by Lewis (2003) states that 'two categories of specimen fabrication methods have been used, these being moulding into a rod or plate and then machining to final dimensions and direct moulding, in a polymeric or metallic mould, without or with external pressurisation, into final dimensions'. Although specimen cross sections are generally made to provide rectangular or circular shapes, geometry of test specimens have varied (Lewis and Nyman, 2000). More concern and debate, however, has been reported regarding the effect of the cross sectional shape rather than the geometry of a specimen. Cristofolini et al. (2002), for instance, used a $4 \mathrm{~mm}$ thickness flat dumbbell shape according to ISO 527 'in order to approximate the in vivo cement mantle thickness closely'. Similarly, a different geometry of a $5 \mathrm{~mm}$ thickness flat shaped specimen was earlier suggested by 
Krause et al. (1988) to represent the cement mantle in vivo, but to be used in testing with 'an appropriate testing protocol' they introduced. In contrast, many studies have preferred testing a circular shape according to specific standards such as ASTM F2118. Lewis and Janna (2003), nonetheless, argue that using either shape should be acceptable as the aim is to characterise the material in vitro. Limited discussion, however, has considered the influence of surface preparation on fatigue behaviour of bone cement specimens. Although it has been suggested that mouldable samples would be preferable to machined (Krause et al., 1988; Paravic et al., 1999; Lewis and Nyman, 2000), both sample production methods are being used.

As for the effect of deformation mode on fatigue behaviour of bone cement, there are no particular studies to specifically investigate this factor. Most studies, however, applied only one mode preferring to use uniaxial tension or compression cyclic deformation modes. Tension-tension or tension-compression stress types, in particular, have been reported in the cement mantle in vivo (Lewis and Nyman, 2000). Tensioncompression loading above all can be particularly preferred because this loading, according to Dowling (2007), provides a good indicator of fatigue performance of materials. Due to these considerations, only very few studies have applied other stress deformation modes such as bending rotating or three point bending of bone cement specimens.

As yet, there is no comprehensive study to compare the effect of variations in both shape and surface finish in the presence of any deformation mode and type. Three previous studies have variously compared different cements under the same conditions involving no more than two testing regimes. Paravic et al. (1999) compared the fatigue behaviour of moulded versus machined circular cross sectional samples that were made from a single cement and were subjected to various tension only peak stress levels. They found apparent longer fatigue lives when the samples were moulded. Another study by Lewis and Janna (2003) compared the fatigue behaviour of rectangular and circular cross sectional shapes using only the moulded sample production method for three bone cements. A factor of difference between 2 to 36 times was found from this work. A more recent study by Tanner et al. (2010) tested four bone cements comparing two methods of fatigue testing where the first method tested rectangular moulded samples under single stress level tension only, followed by Weibull analysis, and the second method performed Wöhler analysis of circular machined samples of the same cements under various stress levels. They found that the difference in fatigue lives between the cements was up to a factor of 15 when the moulded rectangular with Weibull analysis was used and only a factor of 2 when the machined circular and Wöhler analysis method was used.

The aim of the current study is to test and compare fatigue behaviour of two different bone cements using two dumbbell sample shapes: half sized rectangular cross sectional according to ISO 527 (hereinafter called rectangular) or circular cross sectional according to ASTM F2118 (hereinafter called circular). Each of these shapes was produced by either direct moulding or machining from oversized cast bars. Single stress level fully reversed tension compression was used and fatigue results of various sample types were compared by means of Weibull statistical analysis. In addition, initiation and propagation of cracks were investigated through comparing the absorbed energy per loading cycle for each sample type by means of hysteresis loops.

\section{MATERIALS AND METHODS}

\subsection{Materials}

Two different high viscosity bone cements were used. SmartSet GHV (containing gentamicin) and DePuy CMW1 (no gentamicin) were produced and supplied by DePuy CMW as powder (40g) and liquid (18.88g) portions. Each cement has a different opacifying filler in its powder to make the cement visible on X-rays. The GHV powder contains $14.37 \mathrm{wt} \%$ zirconium dioxide as an opacifier (9.76wt\% in the mixture) whereas the CMW powder contains $9.1 \mathrm{wt} \%$ barium sulphate instead $(6.18 \mathrm{wt} \%$ in the mixture). Table 1 compares the chemical compositions of the two bone cements (DePuy CMW, 2012).

\subsection{Samples preparation}

The powder and liquid components were vacuum-mixed at room temperature using the CEMVAC mixing system (DePuy CMW, Blackpool, UK) following the manufacturer's instructions focusing in particular on mixing time, waiting time and working time for each cement. Four PTFE moulds were used to produce four types of samples as follows:

1. Rectangular directly moulded dumbbell samples (RDM)

2. Rectangular moulded and then machined dumbbell samples (RMM)

3. Circular directly moulded dumbbell samples (CDM)

4. Circular moulded and then machined dumbbell samples (CMM)

The moulded and machined rectangular samples were made according to ISO 527 - type 1BA (BS, 2012) as shown in Figure 1a whereas the moulded and machined circular samples were made according to ASTM F2118 (ASTM, 2003) as shown in Figure 1b. Both sample shapes have a nominal gauge cross sectional area of approximately $20 \mathrm{~mm}^{2}$. The difference in the two sample 
Table 1 The powder and liquid chemical contents of SmartSet GHV and DePuy CMW1* (from J\&J website [14])

\begin{tabular}{|l|r|r|r|r|}
\hline & \multicolumn{2}{|c|}{ SmartSet GHV } & \multicolumn{2}{c|}{ DePuy CMW1 } \\
\cline { 2 - 5 } Composition of bone cement powder: & \multicolumn{1}{c|}{ \% $/ \mathrm{w}$} & \multicolumn{1}{c|}{ \% $/ \mathrm{w}$} & \multicolumn{1}{c|}{$\mathrm{g}$} \\
\hline Polymethylmethacrylate & 0 & 0 & 88.85 & 35.54 \\
\hline $\begin{array}{l}\text { Methylmethacrylate/ Methacrylate } \\
\text { Copolymer }\end{array}$ & 80.45 & 32.18 & 0 & 0 \\
\hline Benzoyle Peroxide & 0.96 & 0.384 & 2.05 & 0.82 \\
\hline Gentamicin Sulfate & 4.22 & 1.688 & 0 & 0 \\
\hline Barium Sulphate & 0 & 0 & 9.1 & 3.64 \\
\hline Zirconium Dioxide & 14.37 & 5.748 & 0 & 0 \\
\hline Composition of bone cement liquid: & & & & \\
\hline Methylmethacrylate & 97.5 & 18.408 & 98.5 & 18.5968 \\
\hline N,N-Dimethyle-p-toluidine & $\leq 2.5$ & $\leq 0.47$ & $\leq 1.5$ & $\leq 0.282$ \\
\hline Hydroquinone & $75 \mathrm{ppm}$ & $75 \mathrm{ppm}$ & $75 \mathrm{ppm}$ & $76 \mathrm{ppm}$ \\
\hline *The contents represent a 40g unit (40g powder \& $18.88 \mathrm{~g}$ liquid) & &
\end{tabular}

Figure 1 Test specimen shapes (a) rectangular according to ISO 527 and (b) circular according to ASTM F2118 (units in mm)
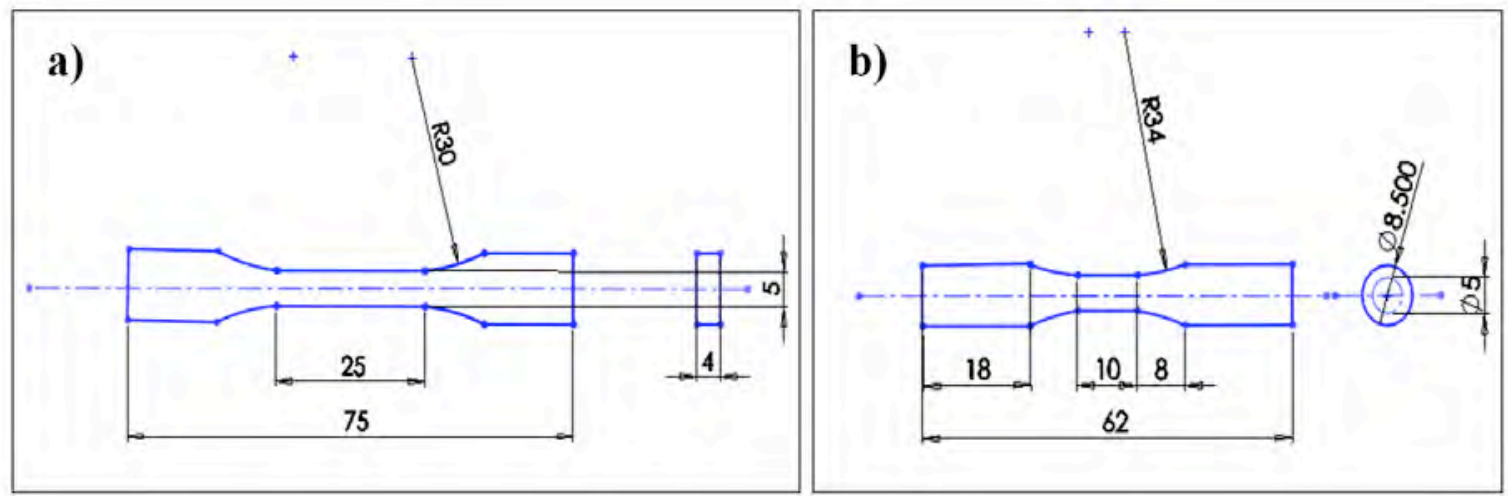

Table 2 Sample shapes and variations in cross and surface areas and surface area to volume ratios

\begin{tabular}{|l|r|r|r|rr|}
\hline & $\begin{array}{l}\text { Gauge cross } \\
\text { sectional area } \\
\left(\mathbf{m m}^{\mathbf{2}}\right)\end{array}$ & $\begin{array}{l}\text { Surface area of } \\
\text { gauge section } \\
\left(\mathbf{m m}^{\mathbf{2}}\right)\end{array}$ & $\begin{array}{l}\text { Volume of } \\
\text { gauge section } \\
\left(\mathbf{m m}^{\mathbf{3}}\right)\end{array}$ & $\begin{array}{l}\text { Surface area } \\
\text { to volume } \\
\text { ratio }\end{array}$ \\
\hline Rectangular & 20 & 450 & 500 & 0.9 \\
\hline Circular & 19.64 & 157.14 & 196.43 & 0.8 \\
\hline
\end{tabular}

shapes is important because of the resultant differences in gauge surface areas, gauge volumes and surface area to volume ratios (Table 2).

To produce these samples, the doughy cement mixture was injected during the working time into a mould which was immediately, before reaching the hardening time, pressurised to 50 bar for 20-30 minutes. This was to ensure the formation of the required sample shapes with limited porosity and to eject the excess cement. The samples were then removed from the mould and incubated at $37^{\circ} \mathrm{C}$ for 24 hours to guarantee initial completion of the polymerisation process. The oversized rectangular and circular samples were machined to the final shape and, along with the mouldable samples, were initially assessed visually under strong light for porosity. Specimens that had pores greater than $1 \mathrm{~mm}$ in diameter were defined as 'macro-pores' and less than $1 \mathrm{~mm}$ as 'micro-pores' (Cristofolini et al., 2002; BialoblockaJuszczyk et al., 2008). Accordingly, samples with large pores $(>1 \mathrm{~mm})$ were rejected. This assessment hypothesis has been criticised because it "will result in comparisons of limited clinical relevance' (Prendergast et al., 2002). However, it is argued that the cross sectional areas of the samples are too small and directly exposed to the experimental fatigue stress; whereas in vivo the stress is distributed over the larger area of the cement mantle. The basic aim is to fatigue test the cement material not 


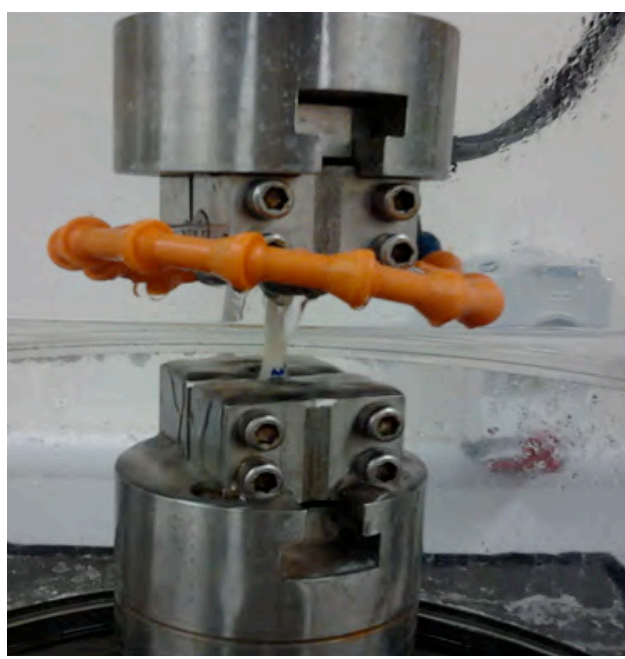

Figure 2 - Close up of a specimen fully covered by saline spray during testing

the specimens themselves since the large voids will occupy significant ratios of the gauge volumes, resulting in earlier failure in a test sample compared to the in vivo mantle. As for the opinion that this porosity rejection consideration is not relevant to the real applications in vivo, the effect of porosity on fatigue strength has already been reported to not match when it comes to comparing the in vivo and in vitro findings (Janssen et al., 2005, Ling and Lee, 1998). After porosity assessment, all samples were soaked in $0.01 \mathrm{M}$ phosphate buffered saline (PBS) at $37^{\circ} \mathrm{C}$ for a period of 1-6 weeks prior to testing. It is recommended that specimens should continually be maintained in the PBS solution between 7 and 60 days (ASTM, 2003).

\subsection{Mechanical testing}

The samples were subjected to fully reversed tensioncompression fatigue testing at a stress of $\pm 20 \mathrm{MPa}$ at $3 \mathrm{~Hz}$. An MTS - 858 Mini Bionix ${ }^{\mathbb{R}} \mathrm{II}$ was used to perform testing and record all the required data according to a specified procedure. During testing, the samples were covered by continuously flowing PBS solution at $37^{\circ} \mathrm{C}$ (Figure 2). The testing data was recorded logarithmically throughout the testing period by the MTS program, that is the first 10 cycles from each hundred until 1000 cycles were recorded, followed by the first 10 cycles from each thousand until 10,000 cycles were recorded and so on. The collected data included recording 10 force and displacement data points for each tensile-compressive loading cycle. At least 10 samples were tested and accepted for each sample type of each cement to have sufficient samples to perform the two parameter Weibull analysis. Lewis and Sadhasivini (2004) estimated the minimum adequate number of bone cement samples in fatigue testing to be either 7 or 11 samples for the two and three parameter
Weibull distributions respectively. Samples that were tested and subsequently found to have macro pores $(>1 \mathrm{~mm})$ in their fracture surfaces, which could not be detected in the initial porosity assessment before testing, were excluded from the analysis.

\subsection{Weibull analysis}

Fatigue testing data was investigated using Weibull analysis to compare the fatigue behavior of the four sample types. The number of cycles to failure of the samples in each set were ranked in ascending order to plot the logarithm of life data $\left(\mathrm{X}=\ln N_{f}\right)$ against Weibull reduced variate $(\mathrm{Y})$ that is determined using equation 1 which requires calculating the cumulative probability of failure $P\left(N_{f}\right)$ using Bernard's approximation (equation 2) where $i$ is the sample rank in a particular population and $n$ is the total number of samples in the population $(i=1$, $2,3, \ldots, n)$. Fatigue results for all sample types were subsequently presented according to their cement formulation on two comparable graphs to obtain four two-parameter Weibull relationships (regression lines) for each cement.

$$
\mathrm{Y}=\ln \ln \left[\frac{1}{1-P\left(N_{f}\right)}\right]
$$

Where, $P\left(N_{f}\right)=\frac{i-0.3}{n+0.4}$

The two-parameter Weibull distribution relationship is given in equation 3 (Hertzberg, 1996; Askeland et al., 2011). The exponential form of this function is given in equation 4 (Weibull, 1951, 1961; Shigley and Mischke, 1989; Danzer et al., 2007; ASTM, 2008; Soh Fotsing et al., 2010).

$$
\begin{aligned}
& \ln \ln \left[\frac{1}{1-P\left(N_{f}\right)}\right]=b \ln \left(N_{f}\right)-b \ln \left(N_{a}\right) \\
& P\left(N_{f}\right)=1-\exp \left[-\left(N_{f} / N_{a}\right)^{b}\right]
\end{aligned}
$$

Where, $P\left(N_{f}\right)$ is the probability of failure after $N_{\mathrm{f}}$ stress cycles, $N_{\mathrm{a}}$ is the characteristic fatigue life (also known as the scale parameter) and $b$ is the Weibull modulus (also known as the shape parameter). The importance of equation 3 is that the left hand side of the equation represents the Weibull variate where the higher this number, the greater the fatigue performance. The right hand side of the equation is dependent on the variables $b$ and $N_{a}$. The Weibull modulus is a measure of skewness of data away from a certain optimal range (normally $3<b<4$ ) during which "approximate symmetry is obtained along with a good approximation to the normal distribution" (Shegley and Mischke, 1989) and that "large $b$ 's skew the distribution to the left and small $b$ 's skew the distribution to the right " (Shegley and 
Mischke, 1989). If all $b$ values are below this range, the dispersion of the $N_{f}$ data is relatively measured so that the higher $b$ value the less the dispersion. The scale parameter $N_{a}$ is obtained as the value below which $63.2 \%$ of the $N_{f}$ results lie. Once $b$ and $N_{\text {a }}$ were obtained, the fatigue performance index $I$ was calculated for each data set according to equation 5 , introduced originally by Britton et al. (1990) when they investigated the shear bond strengths of dental ceramics. This approach has been reported to measure the fatigue performance of bone cement in several studies such as Dunne et al. (2003), Lewis (1999a and 1999b) and Janna et al. (2005). It is worth mentioning that the Weibull mean has similarly been used as an indicator, along with or, instead of $I$ in other studies as Lewis and Janna (2003), Lewis (1999a) and Lewis et al. (2003). The results in this study were compared according to the $I$ indicator and, meanwhile, to see if there is any significance of variations in the fatigue results, the cycles to failure data of relevant testing sets were compared using other statistical approaches, as appropriate, including Student's t-test and the analysis of variance (ANOVA).

$$
I=N_{a} \sqrt{b}
$$

\subsection{Analysis of the hysteresis behaviour}

To provide deeper analysis to the fatigue results, hysteresis loops were used to compare the absorbed energy and apparent modulus per loading cycle for all sample types. Weibull analysis considers only the fatigue life (i.e. the last recorded load cycling data point before failure), but provides no clear indications of how cracks initiate and propagate. The idea of using the absorbed energy concept is built on what has widely been reported regarding the relation between the fatigue crack growth and the hysteresis energy. This energy based criterion is generally divided into two categories depending on which hypothesis is being considered: the amount of absorbed energy is constant and independent of the number of cycles to failure or the amount of absorbed energy changes as the cycles to failure progress (Ellyn, 1997). To simplify the process, it is supposed that 'a fatigue crack growth can be thought of as resulting from energy dissipation (or energy absorption) in the plastic zone' (McCartney, 1996). The change in energy absorption, however, is theoretically attributed to the creation of new free surfaces within the crack zone (Milella, 1999), reflecting the process of crack initiation and propagation. The general relation between the change in absorbed energy and apparent modulus is that the increase in energy follows the same trend as the decrease in moduli (Hoppel and Pangborn, 1994).

To compare the fatigue damage in samples according to these assumptions, the data of a 'typical sample' from each testing group, that is the one closest to the median cycles to failure, was selected to compare a total of 8 median samples. The force and displacement data of these samples was collected as explained above and was converted into stress-strain after selecting data points of certain loading cycles throughout testing so that the 10 data points of the $10^{\text {th }}, 100^{\text {th }}, 1000^{\text {th }}, 5000^{\text {th }}, 10000^{\text {th }}$, $20000^{\text {th }}, 50,000^{\text {th }}$ cycles were included in the analysis. To ensure observing the crack growth behaviour adequately before failure, cycles of the reversing counter positions 5, 100 and 1000 were also considered. Each point of the 10 data points of each of these cycles includes calculating the instantaneous stress and strain. It should be clarified that two different correction factors were applied into the strain results depending on the sample shape. This is due to the reality that the two sample shoulders between the grips and the gauge section are included in testing and the effect of these shoulders on the results must be excluded. These factors were determined using integral calculations and found to be 0.667 and 0.412 for the rectangular and circular samples respectively. Multiplying the initial strain results by these factors will provide the approximate strain occurred in the gauge sections. Once this correction has been considered, the absorbed energy during each of the selected cycles, represented by the area inside the hysteresis loops (Figure 3), was estimated using the Trapezoidal Integration Rule by means of Microsoft Excel spreadsheets. Additionally, the secant modulus at the same cycle was calculated, which is equal to the slope of the straight line that connects the highest and lowest points in the loop. In order to obtain plots of cycles to failure against either absorbed energy per loading cycle and secant moduli, the calculation of the energy and modulus were performed starting from the $10^{\text {th }}$ fatigue loading cycle (loop 1 in figure 3 ) followed by all the other examined cycles and ending with the $5^{\text {th }}$ cycle before failure (loop 2 in figure 3 ).

\subsection{Comparison of fracture surfaces}

Initial investigation of fracture surfaces were performed under an optical microscope. Scanning electron micrographs of the fracture surfaces of the eight median cycles to failure samples were also compared. The aim was to verify if there are any distinct features associated with particular sample types in terms of fatigue fracture behaviour.

\section{RESULTS}

\subsection{Weibull analysis}

The fatigue testing results for different sample types comparing these for SmartSet GHV and DePuy CMW1 are presented in Figure 4 plotting the natural logarithm of cycles to failure against Weibull number. The exclusion of invalid samples according to the macro 


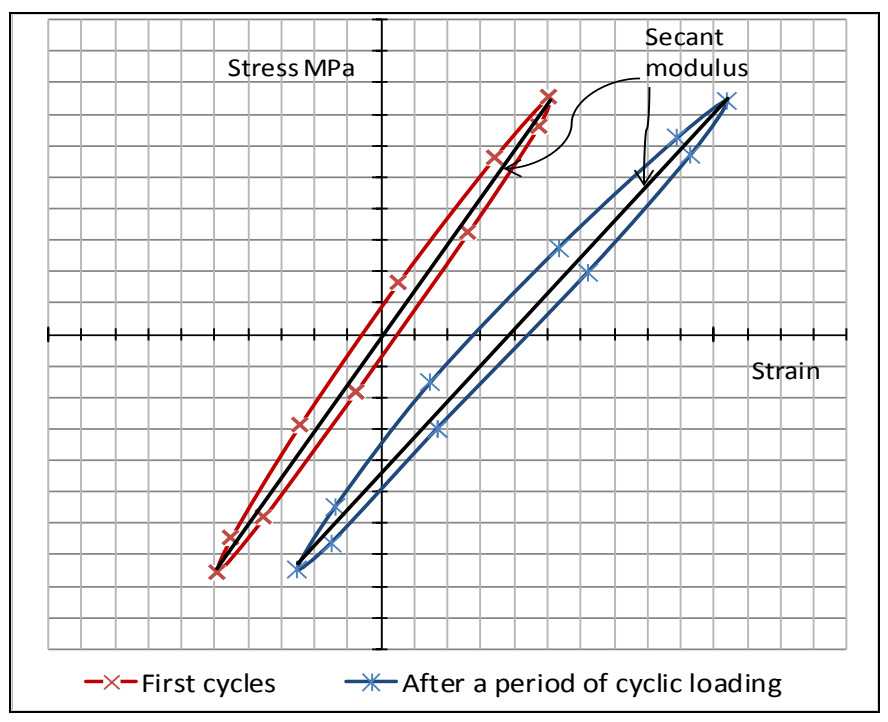

Figure 3 A schematic of the possible change in absorbed energy and modulus throughout testing (from actual test results)

porosity assessment led to an overall average of $23 \%$ of samples to be discarded, approximately two thirds of which occurred after testing. Table 3 summarises the calculated values of the two Weibull parameters; namely the shape parameter or Weibull modulus $(b)$ and the characteristic fatigue life parameter $\left(N_{a}\right)$. The table also shows the calculated values of the fatigue performance index $(I)$ for each testing set which depends on the two parameters values. Due to the fact that this work considers the effect of three parameters simultaneously; namely, sample shape, surface roughness and cement composition, the results presented in Figure 4 and Table 3 make it possible to assess the effect of a particular parameter and compare it to another, depending on the Weibull functions and the fatigue performance indices. For example, the effect of the shaping parameter can be compared for samples that were made from SmartSet GHV for a specific surface production method. In this case, for the moulding conditions, the $I$ index is double for the circular ( $I=75,909$ cycles) compared to the rectangular $(I=37,088$ cycles $)$. Considering the machining conditions only, however, the fatigue indices are almost equal for the circular and rectangular shapes ( $I=14,765$ and 15,678 cycles respectively).

In general and regardless of bone cement type, moulded specimens showed significantly longer fatigue lives compared to the machined specimens $(p=0.00002)$. The results showed 2.5 to 5.5 times greater fatigue performance indices for the moulded specimens $(I=$ $37,088,42,393,75,909 \& 112,970$ cycles) compared to the machined specimens $(I=15,709,19,678,13,346 \&$ 31,235 cycles). The greatest performance is associated, in particular, with the circular moulded specimens $(I=$ $75,909 \& 112,970$ cycles). Compared to their rectangular counterparts $(I=37,088 \& 42,393$ cycles $)$, these circular samples achieved 2 to 2.7 times longer fatigue lives.

Variations in the performance of various samples were also found to depend on the bone cement formation. On the one hand, for the rectangular shape, either moulded or machined, there were no significant differences between the fatigue lives of the SmartSet GHV and DePuy CMW1 samples $(I=42,393 \& 19,678$ cycles and $I=37,088 \& 15,709$ cycles respectively with $p$-value of $0.125)$. There was no evident effect of production method on the fatigue of this rectangular sample shape. On the other hand, however, significant differences in results were determined between the four sets of the circular shape $(p=0.001)$. The circular moulded DePuy CMW1 samples showed 1.5 times greater fatigue index ( $I=112,970$ cycles $)$ and the circular machined 2.3 greater fatigue index $(I=31,235$ cycles $)$ compared to the comparable SmartSet GHV samples $(I=75,909$ \& 13,346 cycles respectively). These results also indicate that, contradictory to the rectangular samples, the effect of production method of circular samples on the fatigue can vary depending on formulation. From Figure 4 and comparing the samples of both cements with regard to production method only, it can also be seen that samples that were produced by direct moulding showed similar fatigue behaviour trends; whereas the samples produced by machining showed the reverse trend where the rectangular machined samples performed better with the Smartset GHV gentamicin and vice versa with the DePuy CMW1.

In terms of dispersion in fatigue results, all the Weibull moduli ( $b$ values) obtained in this work were clearly below the symmetry range. The Weibull distributions skewed to the right for 6 of the 8 testing sets with $b>1$. One testing set (RMM SmartSet GHV) provided exponential distribution $(b=1)$ and only one set $(\mathrm{CMM}$ SmartSet GHV) provided increasing failure probability $(b<1)$.

\subsection{Hysteresis energy and stiffness}

The calculated results from the stress-strain data are presented in figure 5. Generally, similar absorbed energy and stiffness behaviour were seen among the four sample types made from DePuy CMW1 (Figures 5b and 5d) regardless of the differences in fatigue lives. For the four sample types made of SmartSet GHV, obvious variations in the hysteresis energies and moduli can be seen (Figures 5a and 5c), indicating variations in crack propagation among these samples with the highest crack growth speed associated with the machined samples and lower speed for the moulded samples. Comparing the two cements, the fatigue cracks initiate earlier and propagate faster in the SmartSet GHV samples. 


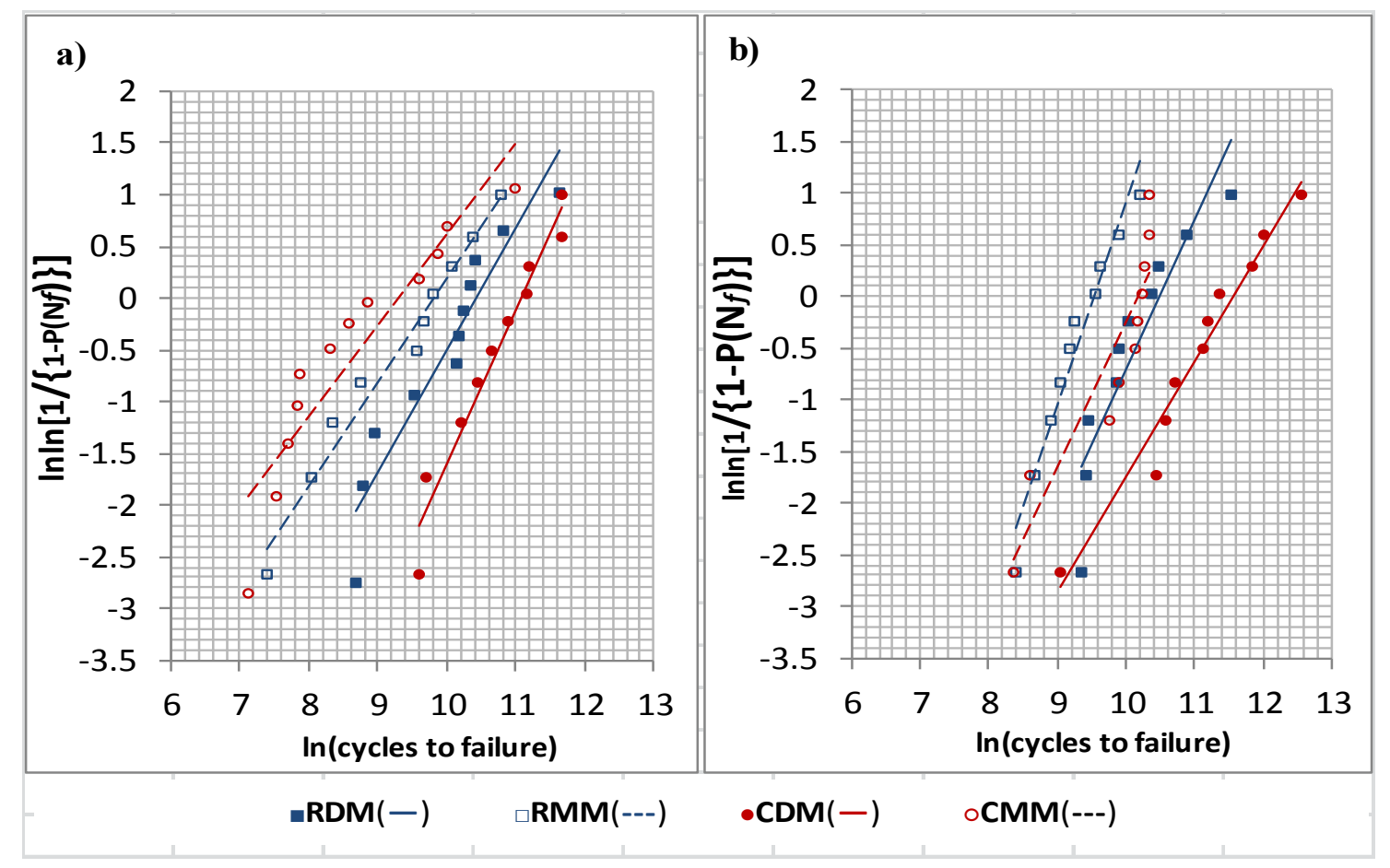

Figure 4 Plots of the two-parameter Weibull relationships showing variations in fatigue behaviour of four different sample types of (a) SmartSet GHV and (b) DePuy CMW1bone cements

Table 3 Summary of the determined values of the shape and characteristics Weibull parameters and resultant fatigue performance index*

\begin{tabular}{|l|r|r|r|r|r|r|}
\hline \multirow{2}{*}{ Sample type } & \multicolumn{3}{|c|}{ SmartSet GHV } & \multicolumn{1}{|c|}{ DePuy CMW1 } \\
\cline { 2 - 7 } & $\boldsymbol{b}$ & $\boldsymbol{N}_{\boldsymbol{a}}$ & $\boldsymbol{I}$ & $\boldsymbol{b}$ & \multicolumn{1}{c|}{$\boldsymbol{\boldsymbol { N } _ { \boldsymbol { a } }}$} \\
\hline RDM & 1.176 & 34,200 & 37,088 & 1.447 & 35,242 & 42,393 \\
\hline RMM & 1.004 & 15,678 & 15,709 & 1.963 & 14,045 & 19,678 \\
\hline CDM & 1.316 & 66,171 & 75,909 & 1.116 & 106,938 & 112,970 \\
\hline CMM & 0.817 & 14,765 & 13,346 & 1.403 & 26,370 & 31,235 \\
\hline
\end{tabular}

$* N_{a}$ and $I$ in cycles

\subsection{Topography of fracture surfaces}

The microscopic view of the fracture surfaces showed similarity between the samples within the same cement (Figure 6). Generally, the SmartSet GHV surfaces were rougher compared to the DePuy CMW1 surfaces. It seems that the cracks start from many points on the circumference of the rectangular or circular fracture surfaces, growing towards the centre where the fracture growth rate is affected by the sample type and cement composition. The side view of the fracture surfaces of randomly selected samples (not shown) led to the conclusion that the 'disconnection topography' between the upper and lower fracture surfaces of a sample is affected by both sample type and cement composition.

\section{DISCUSSION}

The results have shown significant variations in fatigue behaviour. Test materials have revealed up to 5.5 times greater fatigue performance when testing samples produced by direct moulding rather than moulding and machining ( $p=0.00002)$. The longer lives of the moulded samples can be attributed to a few causes. One reason, which has also been reported by Paravic et al. (1999), is that machining of cast rods leads to removal of the outer layer of the material which exposes the internal pores, hence increasing the surface initiation of fractures. Similar to the effect of machined surface porosity, polymers in general have a chance to form free radicals during machining leading to the breaking of bonds at certain depths (Backman and Devries, 1969) 


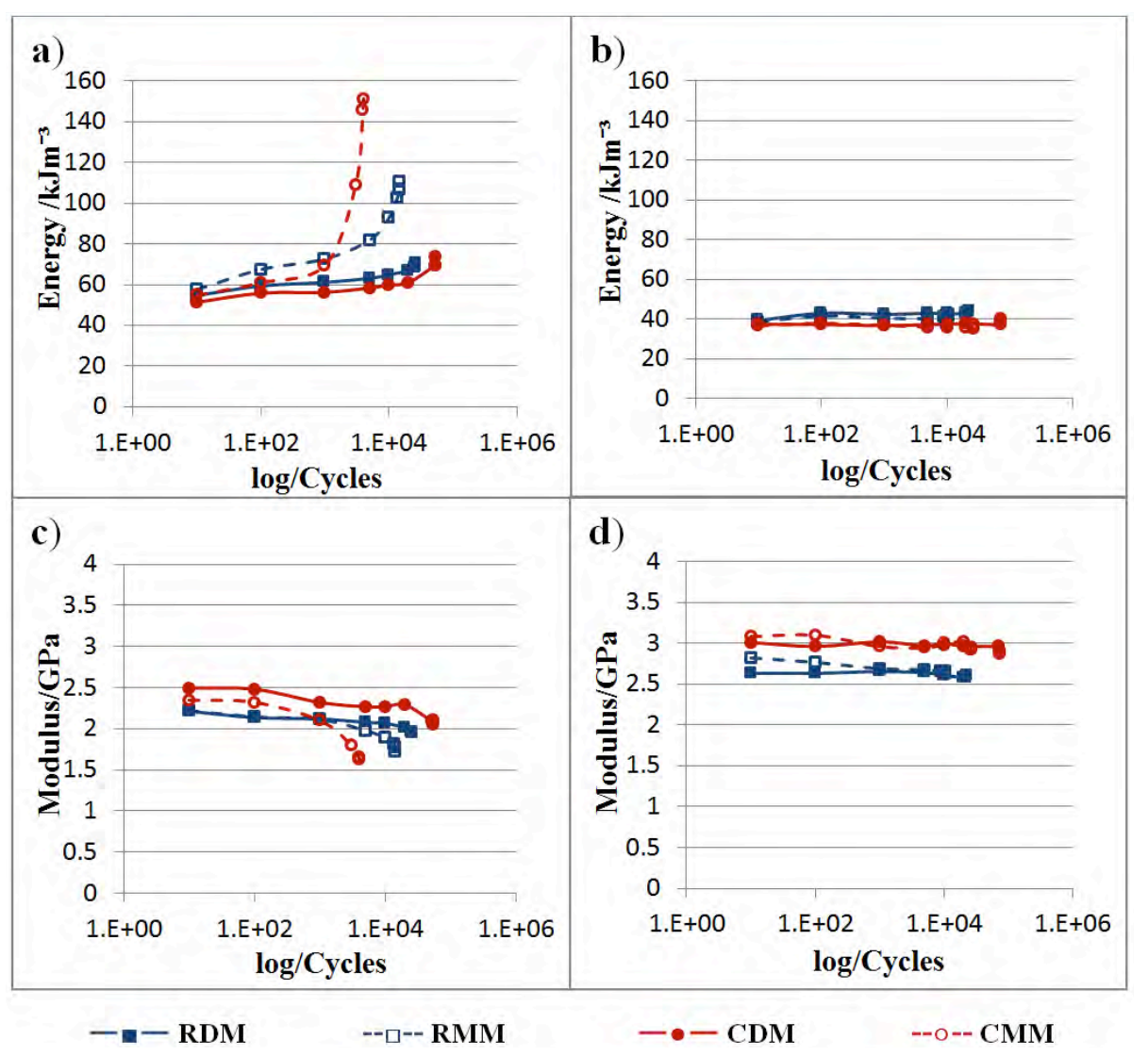

Figure 5 Comparison of absorbed energy and secant modulus behaviours throughout testing for (a and c) SmartSet GHV cement and (b and d) DePuy CMW1 cement

which can alter the tensile and fatigue characteristics. Interpreting the results according to this concept will certainly mean that more potential crack initiation sites existed on the machined samples leading to earlier failure. When moulded, the effect of the degree of interaction between the mould and the bone cement material has been reported by Tanner et al. (2010), however, compared to the machining, it provides less severe surface fracture initiators.

Within the same production method, circular moulded samples provided greater fatigue performance, including the two cement materials, compared to the rectangular counterparts $(\mathrm{p}=0.003)$. Comparing the circular to the rectangular samples produced by machining, however, provided opposite behaviour in results as can be seen in Figure 4. Statistically, however, there is no importance of these variations between the four testing sets within this production method $(\mathrm{p}=0.27)$. Overall, longer fatigue lives were more likely to obtained when testing circular rather than rectangular samples. Similar findings, using different bone cements, have been published particularly when comparing circular moulded against rectangular moulded (Lewis and Janna, 2003) and circular machined against rectangular moulded specimens, but at different fatigue stress types (Tanner et al., 2010). This trend in behaviour can be due to a combination of reasons.
Rectangular samples have nearly three times larger nominal gauge surface area compared to the circular ones (450 $\mathrm{mm} 2$ and $157 \mathrm{~mm} 2$ respectively). This is important since the rectangular specimens have potentially more surface pores increasing the number of crack initiation sites. It is necessary to consider also the effect of the difference between the two sample shapes in terms of surface area to volume ratios. The nominal ratios are 0.8 and 0.9 for the circular and rectangular samples respectively, providing a minimal advantage in crack resistance for the circular specimens. Another reason is that rectangular specimens have sharp corners forming stress concentration regions along the gauge length, whereas the circular samples are free of corners. A few of the rectangular moulded samples, in particular, had accumulations of small pores at these edges, providing weaker regions enhancing the creation of fractures that lead to premature failures. These were less likely to be seen in the rectangular machined samples because they were largely removed during machining. Another cause that has been reported is the residual monomer in each sample shape and its possible variation due to the degree of polymerisation. Lewis and Janna (2003) found that the residual liquid monomer content for the circular specimens can be about $10 \%$ lower than that for the rectangular samples. The study concluded 


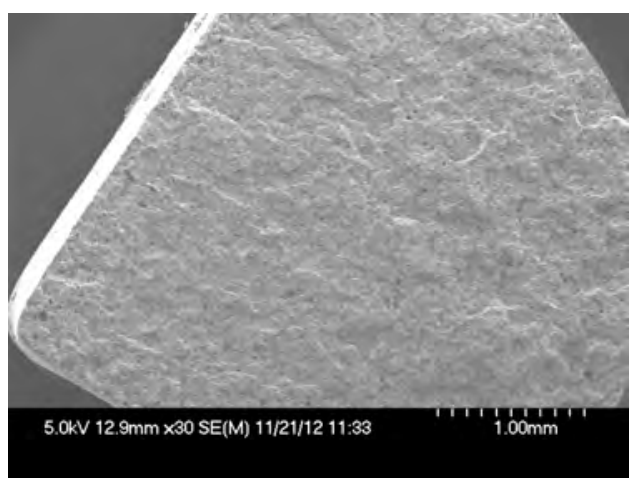

a) RDM

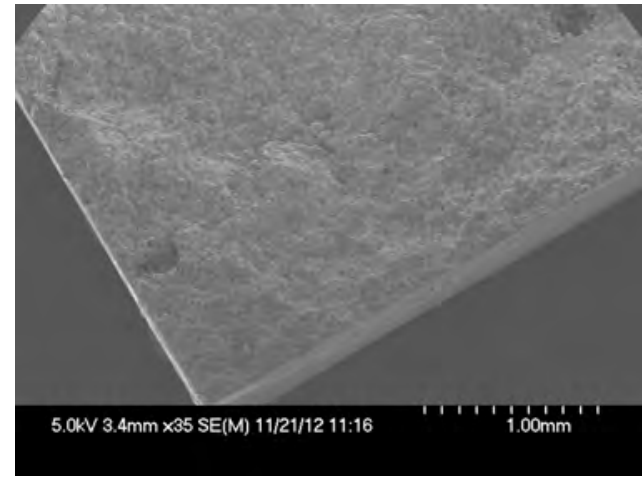

c) $\mathrm{RMM}$

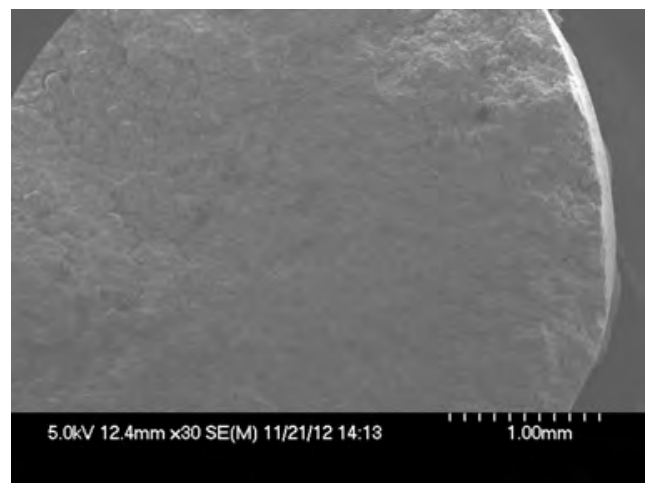

e) $\mathrm{CDM}$

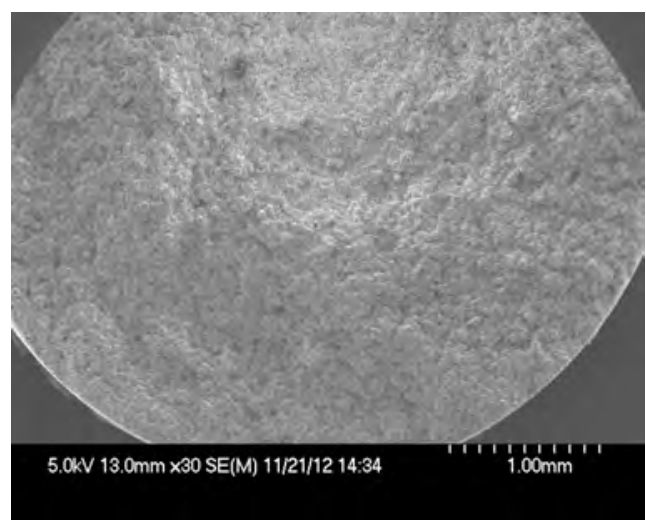

g) $\mathrm{CMM}$

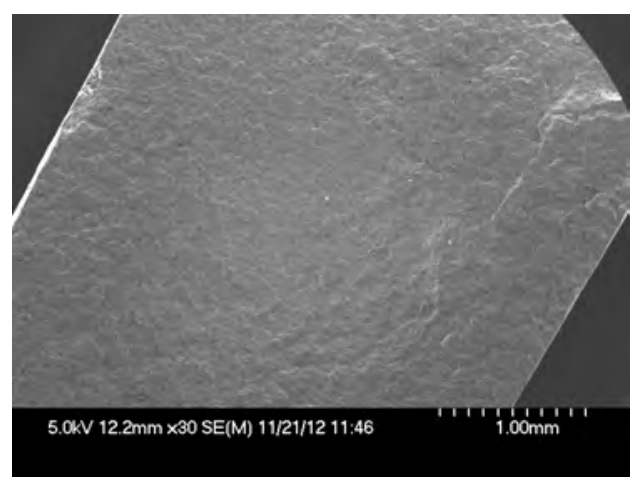

b) RDM

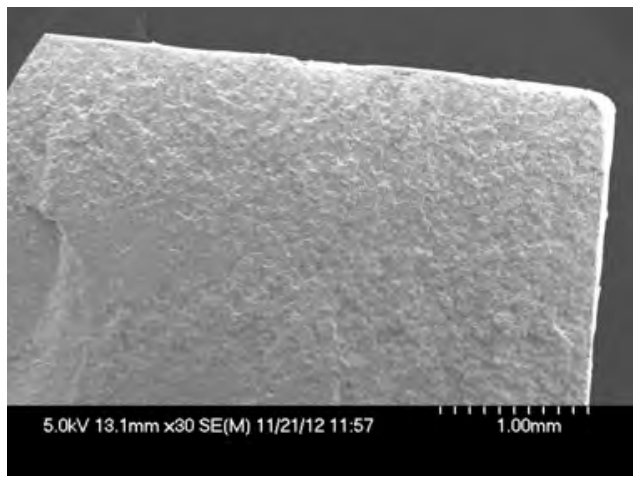

d) $\mathbf{R M M}$

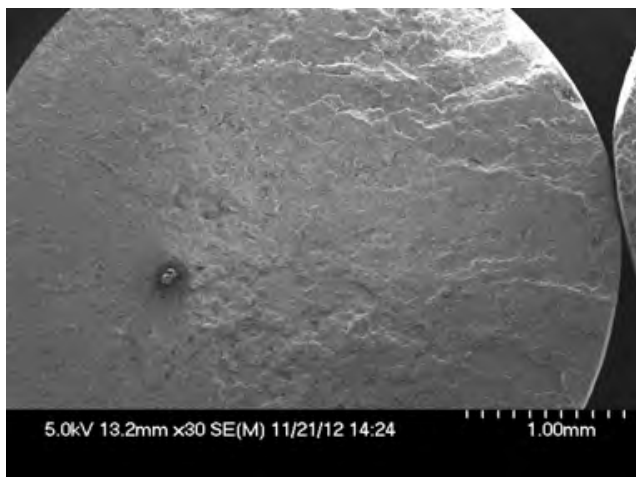

f) $\mathrm{CDM}$

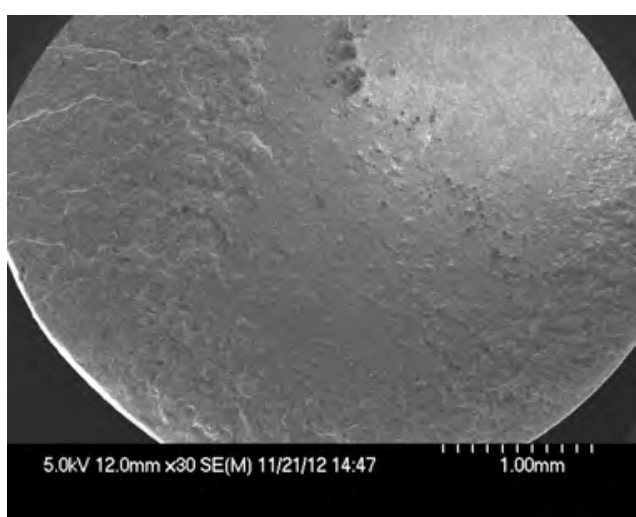

h) CMM

Figure 6 SEM of fracture surfaces of the median samples of different sample types of (a, c, e \& g) SmartSet GHV and (b, d, f \& h) DePuy CMW1 showing ( $\&$ \& ) rectangular directly moulded, (c \& d) rectangular machined, (e \& f) circular directly moulded and $(\mathrm{g} \& \mathrm{~h})$ circular machined. 
that the higher crystalline structure correlated to the circular shape led to the greater fatigue performance. Among the two cement formations (Figure 4), similarity in behaviour has emerged between each moulded shape in the two materials $(p=0.46$ and 0.16 for rectangular and circular respectively). Machined samples in the two materials behaved in a reverse way, yet no significant difference $(p=0.27)$ of these variations was found when comparing the machined sets against each other. Nevertheless, a critical $p$-value $(p=0.05)$ was determined when comparing the circular machined sets of the two cements. To investigate this indistinct correlation further, the two sets can be evaluated according to the fatigue performance index. The weakest sample type was the circular machined SmartSet GHV providing clearly lower fatigue index $(I=13,346$ cycles $)$ compared to its DePuy CMW1 equivalent $(I=31,235$ cycles). As these two sample sets were produced, machined and tested in the same manner, the difference in results can only be due to the variations in chemical structure and additives. The former samples contain $9.76 \mathrm{wt} \%$ of zirconium dioxide and $2.87 \mathrm{wt} \%$ gentamicin whereas the latter set contains only $6.18 \mathrm{wt} \%$ of barium sulphate. When considering the densities of PMMA and the opacifiers, this leads to volume contents of $1.70 \mathrm{vol}$ $\%$ for barium sulphate and 2.20 vol $\%$ for zirconium dioxide. After machining, therefore, the outer surface of the GHV samples had greater chance for the additives to arise that in some cases are surrounded by pores or voids increasing the crack initiations. This aspect might similarly be behind the trend between the fatigue lives of the rectangular machined samples of the SmartSet GHV and DePuy CMW1 though it is not statistically significant $(I=15,709$ and 19,678 cycles respectively and $p=0.2$ ).

Having discussed some possible factors that can variously affect fatigue behaviour of bone cement depending on sample shape, production method and/or chemical structure, it is worth studying what factors have meanwhile controlled the variation in fatigue results within one testing group. The highest Weibull modulus value $(b=1.963)$ associated with the DePuy CMW1 rectangular machined samples showing the lowest variation in results. This correlation, however, is reasonably at low performance of cycles to failure ( $I=19,678$ cycles). That means, in this group, there is no specific factor seemed to have obviously affected one sample rather than the others. The greatest variation in results has been found with the GHV circular machined samples $(b=0.817)$. This category has also shown the lowest fatigue index ( $I=13,346$ cycles) as discussed above. Nine out of 13 samples failed particularly early, in the range between 1000-7000 cycles. When investigating the fracture surfaces of these samples and comparing them to the equivalent GHV circularly moulded samples $(I=75,909)$, similar amorphous structure of the fracture surfaces could be seen with the exception that the topography and circumference of the fracture surface for the machined samples seemed to be rougher than for the moulded ones. This result is presumably because machining process has led to the exposure of more crack initiators.

The investigation of crack behaviour through the hysteresis loops has largely clarified the process of crack initiation and propagation in all sample types. As can be seen in Figure 5, the trends of the increase in absorbed energy and reduction in modulus (stiffness) are similar for DePuy CMW1 samples and varied for SmartSet GHV. Focusing on DePuy CMW1 results only, it can be understood that cracks remained steady with no obvious increase in growth until the last 1000 cycles (Figures $5 \mathrm{~b}$ and 5d). Differently, however, the absorbed energies and moduli for the SmartSet GHV samples showed substantial variations in results (Figures $5 \mathrm{a}$ and $5 \mathrm{c}$ ). In general, for this cement, the cracks initiate earlier and grow more rapidly than those in the DePuy CMW1 cement. This propagation of fatigue cracks is significantly faster with the SmartSet GHV machined samples compared to the moulded ones. Although this approach could substantially explain the variations in fatigue results among samples made of the SmartSet cement, the similarity and stability shown in crack behaviour amongst the DePuy CMW1 samples make it difficult to interpret the fatigue results of these samples in the light of the energy absorption concept. However, it does indicate that cracks in the DePuy CMW1 cement provide superior resistance to fracture growth within the fatigue testing period until a point close to the failure where the crack increases rapidly. This failure, however, as the fatigue life results showed, occurs earlier in the machined samples regardless of the apparent similarity in crack growth behaviour. That is to say, the higher increase in absorbed energy or reduction in modulus in one sample type compared to another does not constantly mean the former type provides shorter fatigue life. For example, the CDM samples made of SmartSet GHV showed clearer increases in energy and decreases in modulus compared to the RMM and CMM samples made of DePuy CMW1, while the fatigue life results of the former cement set were superior.

\section{CONCLUSIONS}

- This study has provided an emphasis that greater fatigue performance is achieved when moulded samples are used in testing. It has meanwhile validated the findings of Lewis and Janna (2003) that circular moulded samples, in particular, provide longer fatigue lives than rectangular moulded samples [I ratios (circular : rectangular) $=2$ for SmartSet GHV and 2.7 for DePuy CMW1, $p=0.003]$.

- It has been shown that the effect of sample shape and production method themselves can be influenced by the type of bone cement used. Although no significant differences have been observed between the rectangular samples in 
both cements [I ratios (DePuy CMW1: SmartSet GHV) $=1.13$ for moulded and 1.27 for machined], clearer variations in fatigue behaviour have been found between the circular samples of the two cements [I ratios (DePuy CMW1: SmartSet GHV) $=1.5$ for moulded and 2.4 for machined).

- Machining of samples can variously affect the outer surface of the sample depending on the amount and type of particles included in the cement matrix, leading to the presence of these elements on the surface creating more crack initiation sites.

- The investigation and analysis of the hysteresis loops, as increases in absorbed energy and reductions in secant modulus, have provided important indications of fatigue crack initiation and growth. Sample shape and production method have been found to significantly alter crack behaviour in the SmartSet GHV cement, whereas these variations were less conspicuous with the DePuy CMW1 cement.

- Overall, it is emphasised, when comparing fatigue results reported in the literature, variations in testing regimes must be considered.

\section{ACKNOWLEDGEMENTS}

The authors thank all those who have contributed to achieving this work. Appreciation is particularly to the Libyan Government for funding the research and DePuy CMW for providing the bone cement materials and mixing devices.

\section{REFERENCES}

Askeland, D.R., Fulay, P.P., Wright, W.J., 2011. The Science and Engineering of Materials, Sixth ed. Cengage Learning, Stamford.

ASTM Standards (2003) F2118-03, Standard test method for constant amplitude of force controlling fatigue testing of acrylic bone cement materials. American Society for the testing of materials, West Conshohocken, PA, US.

ASTM Standards (2008) C 1239-07, Standard practice for reporting uniaxial strength data and estimating Weibull distribution parameters for advanced ceramics. American Society for the testing of materials, West Conshohocken, PA, US.

Backman, D.K., Devries K.L., 1969. Formation of free radicals during machining and fracture of polymers. $J$. Polym. Sci.: Part A-1. 7, 2125-2134.

Bhandari, V. B., 2007. Design of machine elements. Tata McGraw-Hill, New Delhi.
Bialoblocka-Juszczyk, E., Baleani, M., Cristofolini, L., Viceconti, M., 2008. Fracture properties of an acrylic bone cement. Acta. Bioeng. Biomech.10, 21-26.

Britton, J.C., McInnes, P., Ledoux, W.R., Retief, D.H., 1990. Shear bond strength of ceramic orthodontic brackets. Am. J. Orthod. Dentofac. Orthop. 98, 348-353. BSI Standards (2012) ISO 527-2, Plastics determination of tensile properties; Part 2: test conditions for moulding and extrusion plastics. British Standards Institution, London, UK.

Cristofolini, L., Minari, C., Viceconti, M., 2002. Methodology and criterion for acrylic bone cement fatigue tests. Fatigue Fract. Eng. Mater. Struct. 23, 953-957.

Danzer, R., Supancic, P., Pascual, J., Lube, T., 2007. Fracture statistics of ceramics - Weibull statistics and deviations from Weibull statistics. Eng. Fract. Mech., 74, 2919-2932.

DePuy CMW (2012). DePuy orthopaedic gentamicin bone cements. Available from:

https://www.jnjgatewayifu.com/eLabelingContent/Dpo/ USENG/CMWeifu001 84324.pdf,

[Accessed 29/02/2012].

Dowling, N.E., 2007. Mechanical behaviour of materials: Engineering methods for deformation, fracture, and fatigue. Pearson Prentice Hall, New Jersey. Dunne, N.J., Orr, J.F., Mushipe, M.T., Eveleigh, R.J., 2003. The relationship between porosity and fatigue characteristics of bone cements. Biomater. 24, 239-245.

Ellyin, F., 1997. Fatigue damage, crack growth and life prediction. Chapman \& Hall, London.

Gelb, H., Schumacher, H.R., Cuckler, J., Ducheyne, P., Baker, D.G., 1994. In vivo inflammatory response to polymethylmethacrylate particulate debris: effect of size, morphology and surface area. J. Orthop. Res., 12, 83-92.

Gough, H.J., and Pollard, H.V., 1935. The strength of metals under combined alternating stresses. Proceedings of the Institution of Mechanical Engineers [online], Available from:

http://pme.sagepub.com/content/131/1/3.citation,

[Accessed 07/08/2013].

Hertzberg, R.W., 1996. Deformation and fracture mechanics of engineering materials, forth ed. John Wiley \& Sons, Inc. Canada.

Hoppel, C.P.R., Pangborn, R.N., 1994. The evaluation of fatigue damage in short fiber-reinforced styrene-maleic anhydride, in: Mitchel, M.R., Buck, O. (eds.), Cyclic deformation, fracture, and non-destructive evaluation of advanced materials. American Society for Testing and Materials, Philadelphia, PA.

Janna, S., Dwiggins, D.P., Lewis, G., 2005. A new, reliable, and simple-to-use method for the analysis of a population of values of a random variable using the Weibull probability distribution: Application to acrylic bone cement fatigue results. Bio-Med. Mater. Eng. 15, 349-355. 
Janssen, D., Stolk, J., Verdonschot, N., 2005. Why would cement porosity reduction be clinically irrelevant, while experimental data show the contrary. J. Orthop. Res. 23, 691-697.

Krause, W.R., Grimes, L.W., Mathis, R.S., 1988. Fatigue testing of acrylic bone cement: statistical concepts and proposed test methodology. J. Biomed. Mater Res. Appl. Biomater. 22, 179-190.

Lewis, G., Janna, S., Carroll, M., 2003. Effect of test frequency on the in vitro fatigue life of acrylic bone cement. J. Biomed. Mater. Res. Appl. Biomater. 48, 143149.

Lewis, G., Janna, S., 2003. Effect of test specimen crosssectional shape on the in vitro fatigue life of acrylic bone cement. Biomater. 24, 4315-4321.

Lewis, G., Nyman, J.S., 2000. Toward standardization of methods of determination of fracture properties of acrylic bone cement and statistical analysis of test results. J. Biomed. Mater. Res. Appl. Biomater. 53, $748-$ 768.

Lewis, G., Sadhasivini, A., 2004. Estimation of the minimum number of test specimens for fatigue testing of acrylic bone cement. Biomater. 25, 4425-4432.

Lewis, G., 1999a. Effect of mixing method and storage temperature of cement consitituents on the fatigue and porosity of acrylic bone cement. J. Biomed. Mater. Res. Appl. Biomater. 48, 143-149.

Lewis, G., 1999b. Effect of two variables on the fatigue performance of acrylic bone cement: mixing method and viscosity. Bio-Med. Mater. Eng. 9, 197-207.

Lewis, G., 2003. Fatigue testing and performance of acrylic bone-cement materials: state-of-the-art review. $J$. Biomed. Mater. Res. Appl. Biomater. 66B, 457-486.

Lezarus, M.D., Cuckler, J.M., Schumacher, H.R., Ducheyne, P., Baker, D.G., 1994. Comparison of the inflammatory response to particulate polmethylmeth acrylate debris with and without barium sulphate. $J$. Orthop. Res. 12, 532-541.

Ling, R.S., Lee, A.J., 1998. Porosity reduction in acrylic cement is clinically irrelevant. Clin. Orthop. Relat. Res. 355, 249-253.

Malchau, H., Herberts, P., Soderman, P., Oden, A., 2000. Prognosis of total hip replacement: update and validation of results from the Swedish National Hip Arthroplasty Register 1979-1998. 67 ${ }^{\text {th }}$ Annual meeting of the American Academy of Orthopedic Surgeons, Orlando; Available from:

http://www.shpr.se/Libraries/Documents/AAOS2000NH R.sflb.ashx, [accessed 2/4/2013]

McCartney, L.N., 1996. Stress transfer mechanics: Methods that should be the basic of life prediction methodology, in: Johnson, W.S., Larsen, J.M., Cox, B.N., (eds.), Life prediction methodology for titanium matrix composite. American Society for Testing and Materials, West Conshohocken, US.
Mitchell, W., Matthews, J.B., Stone, M.H., Fisher, J., Ingham, E., 2003. Comparison of the response of human peripheral blood mononuclear cells to challenge with particles of three bone cements in vitro. Biomater. 24, 737-748.

Mott, R.L., 2004. Machine elements in mechanical design. Pearson Education, New Jersey.

Milella, P.P., 1999. A fatigue crack growth theory based on energy considerations: Further developments on small crack behaviour and $\mathrm{R}$ ratio, in: Panontin, T.L., Sheppard, S.D. (eds), Fatigue and fracture mechanics. American Society for Testing and Materials, West Conshohocken, PA.

Paravic, V., Nobel, P.C., Alexander, J.W., Leibs, T.R., Elliot, A., 1999. Effect of specimen preparation on porosity and fatigue life a study of centrifuged and hand mixed cements. $45^{\text {th }}$ Annual Meeting, Orthop. Res. Soc., Anaheim, California.

Prendergast, P.J., Murphy, B.P., Taylor, D., 2002. Letter to editor. Fatigue. Fract. Eng. Mater. Struct. 25, 315316.

Quinn, J., Joyner, C., Triffitt, J.T., Athanasou, N.A., 1992. Polymethylmethacrylate-induced inflammatory macrophages resorb bone. J. Bone. Joint. Surg. $[\mathrm{Br}]$ 74B, 652-658.

Sabokbar, A., Fujikawa, Y., Brett, J., Murray, D.W., Athanasou, N.A., 1996. Increased osteoclastic differentiation by PMMA particle-associated macrophages. Acta Orthop. Scand. 67, 593-598.

Shigley, J.E., Mischke, C.R., 1989. Mechanical Engineering Design, fifth ed. McGraw-Hill, New York.

Soh Fotsing, B.D., Ango, G.F., Fogue, M., 2010. Statistical techniques of sample size estimating in fatigue tests. Int. J. Eng. Technol. 2, 477-481.

Suresh, S., 1998. Fatigue of materials. Cambridge University Press, New York.

Swanson, S.R., (ed.) 1974. Handbook of fatigue testing. American Society for Testing and Materials, Philadelphia.

Tanner, K.E., Wang, J.S., Kjellson, F., Lidgren, L., 2010. Comparison of two methods of fatigue testing bone cement. Acta Biomater. 6, 943-952.

Wang, J.S., Diaz, J., Sabokbar, A., Athanasou, N., Kjellson, F., Tanner, K. E., McCarthy, I.D., Lidgren, L., 2005. In vitro and in vivo biological responses to a novel radiopacifying agent for bone cement. J. R. Soc. Interface. 2, 71-78.

Weibull, W., 1951. A statistical distribution function of wide applicability. J. Appl. Mech. 18, 293-297.

Weibull, W., 1961. Fatigue Testing and Analysis of Results, Pergamon Press, London. 